Психология. Журнал Высшей школы экономики. 2015. T. 12. № 4. С. 165-183.

Обзоры и рещензии

\title{
ЭФФЕКТ ФЛИННА: ОБЗОР СОВРЕМЕННЫХ ДАННЫХ
}

\author{
Е.А. ВАЛУЕВА, С.С. БЕЛОВА
}

\begin{abstract}
Резюме
Статья представляет обзор современной зарубежной литературы по проблематике эффекта Флинна. Эффектом Флинна называется рост показателей по тестам интеллекта на протяжении XX в., его темп оценивается примерно в 3 балла IQ за десятилетие. В статье описываются сущность ЭФ и методы его измерения, метааналитические данные о размерах и стабильности ЭФ между популяциями и во времени. Рассматривается проявление ЭФ на данных решения когнитивных задач, отличных от тестов интеллекта, и в сфере интеллектуальных достижений. Особое внимание уделяется дискуссии о причинах эффекта Флинна. Наиболее популярными являются средовые объяснения эффекта Флинна (улучшение питания, изменения в составе семьи, повышение количества и качества когнитивной стимуляции и т.д.). Вместе с тем существуют объяснения, связывающие эффект Флинна с генетическими изменениями, а также с артефактами, возникающими в процессе измерения интеллекта. Также ставится вопрос о том, означает ли эффект Флинна реальный прирост интеллекта людей. Приводятся данные, согласно которым эффекту Флинна в наибольшей степени подвергаются задания, имеющие наименьшие нагрузки на генеральный фактор интеллекта (фактор g). Одновременно с этим существуют данные, свидетельствующие о снижении генотипического интеллекта. Делается вывод о том, что эффект Флинна связан с изменением структуры когнитивных способностей и приобретением навыков решения задач, требующих оперирования правилами и абстрактными умозаключениями. При этом эффект Флинна маскирует тенденцию снижения генотипического интеллекта. В завершение статьи рассматриваются практические следствия эффекта Флинна в сфере образования, судебного делопроизводства и социальной политики.
\end{abstract}

Ключевые слова: эффект Флинна, интеллект, тесты интеллекта. 


\section{Что такое эффект Флинна}

C начала XX в. тесты интеллекта широко применяются на Западе в академической, военной, профессиональной и даже судебной сферах. Так, со времен Первой мировой войны новобранцы армии США проходят скрининговое тестирование, призванное определить их пригодность к военной службе или к занятию определенных должностей в армии, а в некоторых штатах результат по тесту интеллекта оказывается решающим при принятии решения о возможности применения высшей меры наказания (смертной казни) к подсудимому (McGrew, 2015).

Оценка интеллекта каждого отдельного человека (балл IQ) является продуктом сравнения его индивидуального результата с результатами других людей соответствующей возрастной группы. Интеллект в 100 баллов соответствует количеству заданий теста, которые решают не менее 50\% людей из нормативной выборки (пятидесятый процентиль). Интересно, что в Менсу (в организацию людей с высоким коэффициентом интеллекта) принимают лишь тех, кто показал результат на уровне не ниже 98 процентиля: это соответствует примерно 130 баллам IQ и означает, что лишь два процента людей обладают таким же или более высоким интеллектом.
Несколько первых десятилетий применения тестов интеллекта показали, что нормы (т.е. процент людей, успешно решающих определенное количество заданий в тесте) не остаются неизменными (Merrill, 1938; Tuddenham, 1948). В 1980-х гг. Джеймс Флинн систематизировал накопленные к тому моменту результаты по тестам интеллекта и обнаружил, что в США (Flynn, 1984), так же как и в других странах (Flynn, 1987), люди «умнеют» в среднем на 3 балла IQ за десятилетие. Зафиксированный Дж. Флинном рост показателей по тестам интеллекта на протяжении XX в. получил «официальное» название «эффект Флинна» (ЭФ) ${ }^{1}$ в 1994 г. в книге Р. Хернстайна и Ч. Мюррея (Herrnstein, Murray, 1994).

\section{Как оценивается эффект Флинна}

Для оценки ЭФ исследователи могут использовать разные методы. Один из методов (интраиндивидуальный) предполагает выполнение одними и теми же испытуемыми двух разных тестов: это могут быть разные версии одного и того же теста (Flynn, 2009) или вообще совершенно разные тесты (Trahan, Stuebing, 2014), главное, чтобы нормативные данные по этим тестам были получены в разные годы. ЭФ оценивается как разница в баллах IQ между «старым» (стандартизованным раньше)

${ }^{1}$ Некоторые исследователи (см., например: Rushton, 1999) придерживаются мнения, что более правильным названием для ЭФ было бы «эффект Линна-Флинна», так как в 1982 г. (т.е. за два года до работы Дж. Флинна) Р. Линн опубликовал работу, в которой зафиксировал рост показателей интеллекта в Японии (Lynn, 1982). Термин «эффект Линна-Флинна» указывает на одинаковую значимость вклада двух авторов в привлечение внимания к проблеме роста баллов IQ. 
и «новым» тестами. Чтобы вычислить средний прирост интеллекта в год, эту разницу делят на количество лет, разделяющих даты стандартизации двух тестов.

Другой метод (интериндивидуальный) заключается в сравнении когорт испытуемых, выполнявших один и тот же тест в разные годы. Так, например, в Норвегии (Sundet et al., 2004) или Дании (Teasdale, Owen, 2008) практически все мужчины призывного возраста выполняют набор тестов, измеряющих разного рода интеллектуальные способности: вербальные, числовые, общие. Задания тестов остаются неизменными на протяжении многих лет, что позволяет напрямую сравнивать сырые баллы, полученные разными поколениями испытуемых. В этом случае годовой ЭФ вычисляется как разница в оценках IQ разных поколений, поделенная на количество лет, прошедших между тестированиями. Преимущество этого метода заключается в его простоте, однако необходимость соблюдать подобие выборок на нескольких временны́х срезах накладывает ограничения на возможность его применения.

Метод перекрестного анализа предполагает тестирование одним и тем же тестом людей разного возраста. ЭФ оценивается как разница между средними показателями IQ в двух возрастных когортах. Для получения надежных результатов, однако, важно соблюдать репрезентативность каждой выборки, а также необходимо учитывать возрастные изменения интеллектуальных функций, специфичные для конкретного типа способностей (Meisenberg et al., 2005).

\section{Эффект Флинна: обобщенные данные}

Исследования ЭФ развернулись на данных, собранных во многих странах, и были обобщены в нескольких метаанализах (Fletcher et al., 2010; Pietschnig, Voracek, 2015; Trahan, Stuebing, 2014). Так, в метаанализе Я. Питчнига и М. Ворачека анализировались данные 219 исследований, включающих результаты примерно четырех миллионов человек, тестировавшихся с 1903 по 2013 г. в 31 стране по всему миру ${ }^{2}$. Метааналитические исследования подтверждают существование ЭФ и оценивают его размер от 2.31 (Trahan, Stuebing, 2014) до 2.8 (Pietschnig, Voracek, 2015) балла IQ за десятилетие. Показано, что для флюидного интеллекта ЭФ более выражен, чем для кристаллизованного; что прирост интеллекта на протяжении XX в. хотя и был стабильным, но не был линейным (т.е. ускорялся или замедлялся в определенные десятилетия) (Там же); что ЭФ одинаково выражен в группах с разным уровнем интеллектуальных способностей (Trahan, Stuebing, 2014) и коррелирует с ростом валового внутреннего продукта (Pietschnig, Voracek, 2015). Также выявлена общая тенденция замедления ЭФ в последние десятилетия (особенно

${ }^{2}$ В список анализируемых стран не вошла Россия в связи с полным отсутствием релевантных и адекватных психометрических исследований (Батурин, 2008). 
выраженная для кристаллизованного интеллекта), связанная с появлением так называемого «негативного» эффекта Флинна, наблюдаемого в некоторых европейских (преимущественно скандинавских) странах (Dutton, Lynn, 2013, 2015; Sundet et al., 2004; Teasdale, Owen, 2008).

\section{За пределами тестов интеллекта}

Интересным является вопрос, наблюдается ли ЭФ в областях, отличных от тестов интеллекта. Показано, что ЭФ наблюдается для эпизодической и семантической памяти (Rönnlund, Nilsson, 2009), для объема числовой рабочей памяти при воспроизведении в прямом порядке (digit span forward) (Woodley of Menie, Fernandes, 2015), для знания чисел и стратегий их понимания в дошкольном возрасте (Bocéréan et al., 2003). Также были зафиксированы изменения реальных достижений людей в разных сферах - более раннее развитие мастерства в интеллектуальных играх в онтогенезе, рост количества научных публикаций и патентов (Howard, 2001). Вместе с тем для ряда способностей наблюдаются эффекты, противоположные ЭФ, - замедление простого времени реакции (Woodley et al., 2013), уменьшение объема числовой рабочей памяти при воспроизведении в обратном порядке (digit span bakward) (Woodley of Menie, Fernandes, 2015), снижение продуктивности в формальных пиажеанских операциях (Shayer, Ginsburg, 2009), сокращение темпов инноваций на душу населения и числа «гениев» (Woodley of Menie, Fernandes, 2015).

\section{Причины эффекта Флинна}

В настоящее время большинством исследователей признается, что причины ЭФ кроются скорее в средовых, а не в генетических факторах: показано, что прирост баллов по тестам IQ коррелирует отрицательно с показателями, имеющими высокую генетическую составляющую, - нагрузками на g-фактор, инбредной депрессией и т.д. (Rushton, 1999; Rushton, Jensen, 2010). Такое положение дел названо У. Дикенсом и Дж. Флинном «парадоксом IQ»: высоко наследуемая характеристика (интеллект) претерпевает в течение десятилетий значительные изменения, которые имеют преимущественно средовые причины (Dickens, Flynn, 2001).

Несмотря на это, существуют теории, согласно которым природа ЭФ связана преимущественно с генетическими механизмами. Так, М. Мингрони (Mingroni, 2007, 2014) в качестве объяснения предлагает механизм гетерозиса - увеличения гетерозиготности в сообществе за счет браков, заключаемых между генетически отдаленными популяциями (что стало возможно благодаря повышению мобильности населения в XX в. и уменьшению количества обособленно живущих групп). Гетерозис предполагает «улучшение» свойств индивида за счет перераспределения доминантных и рецессивных признаков. Гетерозис противоположен по своей сути инбредной депрессии, которая повышает гомозиготность и (в случае интеллекта) заключается в снижении умственных способностей детей, чьи родители заключили близкородственные браки (Agrawal et al., 1984; Jensen, 
1983). Одним из аргументов М. Мингрони в пользу развиваемой теории является «правило параллельных изменений» (Mingroni, 2014): рост интеллектуальных показателей в популяции сопровождается изменением других наследуемых характеристик (рост, половое созревание, размеры мозга, личностные черты и т.д.). М. Мингрони рассматривает генетические данные о распределении аллелей у жителей 74 некогда обособленных друг от друга приходов Пармской провинции в Италии. На базе этих данных он строит модель, основанную на допущении, что существуют доминантные гены, ведущие к повышению интеллекта, и рецессивные, не ведущие к нему. Появление в генотипе двух доминантных аллелей повышает интеллект на условную единицу, появление двух рецессивных не изменяет интеллект, а генотип с одним доминантным и одним рецессивным аллелем увеличивает интеллект на величину D, варьирующую от 0.5 до 1. С помощью этой модели М. Мингрони показывает, как изменился бы интеллект итальянцев, если бы они, например, объединились в одно сообщество и стали бы заключать браки «случайным» образом или если бы браки стали заключаться между членами отдаленных друг от друга сообществ. В целом его результаты показывают, что заключение браков более «случайным» образом приводит к эффектам, очень похожим на ЭФ.

Еще одна гипотеза, так или иначе связывающая ЭФ с генетическими (точнее, эпигенетическими) механизмами, - гипотеза геномного импринтинга (Storfer, 1999). Согласно этой гипотезе, рост интеллекта родителей, произошедший в результате стимулирующих воздействий окружающей среды, закрепляется в следующих поколениях в соответствии с принципами эпигенетической наследуемости.

Средовые гипотезы можно объединить в несколько групп. Гипотезы одной группы предполагают позитивное влияние на состояние организма того или иного фактора, вследствие чего возникают благоприятные условия для когнитивного развития. Среди таких факторов оказываются: улучшение пре- и постнатального питания (Lynn, 1990, 2009), увеличение воздействия искусственного света (Williams, 2013), уменьшение патогенного стресса, вызванного инфекционными болезнями (Eppig et al., 2010), понижение уровня свинца в крови (Nevin, 2000) и т.д. Другая группа гипотез включает факторы, связанные с изменением в устройстве семьи - уменьшением ее размера (Zajonc, Mullally, 1997) и увеличением заинтересованности родителей во всестороннем развитии детей (Neisser, 1997). Еще одна группа «средовых» гипотез связана с повышением и изменением качества когнитивной стимуляции - увеличением количества и качества образования (Husen, Tuijnman, 1991; Teasdale, Owen, 2005), обогащением визуального опыта и опыта взаимодействия с современными технологиями (книги с картинками, пазлы, фильмы, компьютеры, планшеты, видеоигры и т.д.) (Greenfield, 1998; Neisser, 1997), повышением сложности окружающей среды (Schooler, 1998).

Многие из названных гипотез находят как подтверждающие, так и опровергающие их данные. Это заставляет исследователей все больше склоняться к мнению о комплексном 
характере причин ЭФ. Так, например, сам Дж. Флинн в попытках преодолеть обозначенный им парадокс IQ (значительные, вызванные средовыми причинами изменения интеллекта, несмотря на его высокую наследуемость) предложил гипотезу генно-средового взаимодействия (Dickens, Flynn, 2001). В ее основе лежит представление о взаимной обусловленности генотипа и среды: более высокий интеллект приводит к выбору соответствующей среды, стимулирующей когнитивные усилия, которые, в свою очередь, ведут к повышению интеллекта и т.д. (индивидуальная мультипликация). Кроме того, предполагается эффект социальной мультипликации, который заключается в том, что уровень интеллекта окружающих людей влияет на уровень интеллекта отдельного человека, а уровень интеллекта отдельного человека (в случае его повышения стимулирующими воздействиями окружающей среды) повышает уровень интеллекта социального окружения и т.д. Согласно У. Дикенсу и Дж. Флинну, средовые воздействия (триггеры роста интеллекта) могут быть самыми разнообразными и необязательно являются одинаковыми для разных индивидов и постоянными во времени. Ключевой момент заключается в том, что средовые изменения «усредняются» и создают константный позитивный средовой эффект, который запускает процессы индивидуальной и социальной мультипликации. Таким образом, по мнению авторов, даже незначительные изначальные средовые изменения могут вызвать значительные изменения интеллекта, несмотря на его высокую наследуемость.
Другая комплексная модель причин ЭФ предложена М. Вудли (Woodley, 2012a). Его гипотеза усилий по когнитивной дифференциации-интеграции (cognitive differentiation-integration effort hypothesis) основана на модели скорости жизненного цикла (life history model Rushton, 1985). Быстрый жизненный цикл предполагает большее количество сексуальных партнеров в течение жизни, бо́льшее количество детей, меньшие усилия, вкладываемые в воспитание каждого отдельного ребенка. Медленный жизненный цикл, наоборот, связан с более стабильными браками, меньшим количеством детей и большими вложениями в их воспитание. М. Вудли предположил, что с точки зрения когнитивных способностей быстрый жизненный цикл связан с когнитивной интеграцией - усилением фактора g за счет одинаковых инвестиций в разные типы способности. Такая стратегия выгодна в ситуациях, которые характерны для быстрого жизненного цикла и требуют координации разнообразных способностей. Медленный жизненный цикл предполагает когнитивную дифференциацию - возрастающую специализацию способностей за счет неравноценных инвестиций. В современном мире существует тенденция к замедлению жизненного цикла в связи с улучшением жизненных условий в целом, снижением непредсказуемости, улучшением гигиены, питания и т.д. Замедление жизненного цикла объясняет ЭФ: происходит когнитивная дифференциация, вследствие чего растут показатели по отдельным способностям, но при этом наблюдается снижение интеркорреляций между 
способностями (Juan-Espinosa et al., 2006; Kane, 2000; Kane, Oakland, 2000; Lynn, Cooper, 1993, 1994).

Помимо генетических и средовых объяснений ЭФ, существует мнение, что рост показателей по тестам может быть артефактом, связанным с приобретением испытуемыми навыков тестирования. В частности, предполагается, что начинают играть роль ставшие более изощренными стратегии угадывания (так называемый эффект Брэнда - Brand et al., 1989). Показано, что, по крайней мере, часть прироста баллов IQ можно объяснить за счет возросшего процента угадывания (Must, Must, 2013; Pietschnig et al., 2013; Woodley et al., 2014). Также есть исследования, показывающие, что применение IRT-анализа может существенно снижать оценки прироста интеллекта в рамках ЭФ (Beaujean, Osterlind, 2008; Beaujean, Sheng, 2010; Shiu et al., 2013).

\section{Действительно ли человечество умнеет?}

Наиболее важный вопрос, касающийся ЭФ: что же на самом деле означает повышение показателей по тестам интеллекта? Стоит ли за ЭФ реальное прибавление количества «ума» или это просто артефакт, связанный с несовершенством измерения? Если происходит реальный рост интеллекта, то умнеют ли люди «вообще» или происходит развитие лишь специальных способностей? Ответ на последний вопрос исследователи часто переформулируют так: «Является ли ЭФ эффектом Дженсена?». А. Дженсеном был предложен метод коррелирующих векторов
(Jensen, 1998), который позволяет выяснить, сколько общей дисперсии c фактором g имеет та или иная характеристика. Эффектом Дженсена называется характеристика, положительно связанная с нагрузками субтестов на фактор g. Так, например, если взять, с одной стороны, вектор нагрузок субтестов на фактор g, а с другой стороны - вектор корреляций результатов по этим субтестам с какой-либо переменной (размером мозга или временем реакции испытуемого), а потом прокоррелировать их между собой, то можно выяснить, в какой степени связаны с g соответствующие переменные. Как правило, высоко связанными с фактором g оказываются переменные, имеющие высокую степень генетической обусловленности - показатели наследуемости и инбредной депрессии субтестов, размер мозга, время реакции испытуемых и т.д. Результаты исследований показывают, что ЭФ, напротив, оказывается «антиэффектом» Дженсена: прирост по тестам интеллекта оказывается отрицательно связанным с их нагрузками на g (te Nijenhuis, van der Flier, 2013; Woodley et al., 2014). Отрицательная связь ЭФ с нагрузками на g означает две вещи: во-первых, ЭФ затрагивает дисперсию способностей, не связанную с g, т.е. в результате ЭФ не происходит роста уровня общего интеллекта; во-вторых, ЭФ имеет средовую природу, так как отрицательно связан с переменной, имеющей высокий показатель наследуемости.

Между тем некоторые исследования показывают, что «истинный» (генотипический) интеллект демонстрирует определенную динамику в 
истории человечества (Woodley, $2012 \mathrm{~b}$ ) и в настоящее время имеет тенденцию к снижению. Генотипический интеллект - это интеллект, который мог бы продемонстрировать человек в оптимальных средовых условиях (Там же). Снижение генотипического интеллекта связывается с дисгенетической фертильностью, т.е. с тенденцией рождения меньшего количества детей у людей с более высоким уровнем интеллекта (Shatz, 2008), а также с ослаблением естественного отбора (Woodley of Menie, 2015). Снижение интеллекта на протяжении XX в., вызванное дисгенетической фертильностью, отдельными исследованиями оценивается от 0.33 до 0.9 баллов за поколение (Woodley, 2012b), а метаанализ показывает, что две причины в совокупности снижают генотипический интеллект на 1.23 балла за десятилетие. Косвенно данные о снижении генотипического интеллекта также подтверждаются наличием антиэффекта Флинна по наиболее связанным с g переменным (время реакции, феномены Пиаже, рабочая память при обратном порядке воспроизведения).

В отличие от генотипического интеллекта, фенотипический интеллект - это интеллект, измеренный в баллах IQ и являющийся результатом развития генетически заданного потенциала в реальных средовых условиях. Именно рост фенотипического интеллекта фиксируется, когда идет речь об ЭФ. Таким образом, дисгенетические эффекты (связанные с g) и ЭФ (не связанный с g) развиваются параллельно, и, вероятно, ЭФ в той или иной степени маскирует снижение генотипического интеллекта (Lynn, Harvey, 2008).
Исследования показывают, что явления, сопровождающие рост интеллекта в рамках ЭФ, во многом сходны с явлениями, наблюдаемыми для тест-ретестового роста баллов IQ, а также в случаях повышения баллов IQ в результате специальных развивающих программ. К таким явлениям относятся: низкая нагруженность прироста по фактору g, тренировка специфичных для конкретного теста когнитивных компонентов, низкий уровень переноса навыка на другие области (что напрямую связано с низкой нагрузкой на g) (te Nijenhuis et al., 2014; te Nijenhuis et al., 2007).

Таким образом, с одной стороны, рост баллов IQ оказывается не связанным с ростом интеллекта в смысле генерального фактора, а с другой стороны, заметна явная динамика интеллектуальных способностей. Эту динамику Дж. Флинн называет «когнитивным прогрессом» (Flynn, 2015): смена когнитивной среды, связанная с научно-технической революцией, стала требовать от людей развития способностей, связанных с абстрактным мышлением и логикой. Развитие этих способностей стало возможным из-за изменений в системе образования, питания, семьи и т.д. Структура когнитивных способностей («склад ума») становится другой (Ушаков, 2011), поэтому в каком-то смысле люди «умнеют» - они становятся все более способными к решению когнитивно-сложных задач, требующих оперирования правилами и абстрактными умозаключениями. Вместе с тем со временем непосредственные причины ЭФ (улучшенное питание и образование, уменьшение размера 
семьи и т.д.) исчерпывают свой ресурс, рост когнитивных способностей достигает «эффекта потолка», поэтому прогноз заключается в том, что ЭФ постепенно будет сходить на нет.

\section{Практические следствия}

ЭФ имеет практические следствия в тех сферах, где психометрический интеллект выступает одним из критериев при принятии решений в отношении конкретных людей. В сфере образования США ЭФ приводит к флуктуациям в определении доли учащихся, которым рекомендованы специальные образовательные программы. Например, в начале 1990-х гг. при переходе к WISC-III доля учащихся с умственной отсталостью (intellectual disability) в два раза превышала значение последних лет применения WISC-R (Kanaya et al., 2003). Такая закономерность проявилась в большинстве американских штатов (Scullin, 2006). При этом категория учащихся с трудностями в обучении (learning disabilities) - распространенным диагнозом, предполагающим интеллект в рамках нормы и нарушение по меньшей мере одного из фонологических процессов, - наоборот, оказалась сокращена (Kanaya, Ceci, 2012). Экономические и социально-психологические следствия подобных колебаний очень значительны (Fitzgerald et al., 2007). Использование устаревших норм может не выявлять всех действительно нуждающихся в специальном образовании в связи с умственной отсталостью. Использование же новых норм, наоборот, может способствовать переоценке их количества и повышать риск социальной стигматизации учащихся в связи с постановкой диагноза и негативных последствий от нерелевантных педагогических интервенций. Ясного универсального решения этой проблемы пока не существует, сформулирована лишь рекомендация к использованию в образовании наиболее современных тестовых норм (Kanaya, Ceci, 2012).

В судебной практике США диагноз умственной отсталости предполагает запрет смертной казни. Шансы обвиняемого избежать ее возрастают, если осуществляется поправка его IQ на устаревание норм на момент тестирования путем вычитания соответствующего прироста баллов. Сегодня позиция экспертного большинства состоит в том, что в случаях решения вопроса о смертной казни такая поправка необходима и справедлива (Cunningham, Tassé, 2010; Fletcher et al., 2010; Flynn, 2006; Gresham, Reschly, 2011; McGrew, 2015; Young, 2012). Однако критика этой практики - как в судебной, так и в образовательной сфере - указывает на ее значительные ограничения. Недостаточно эмпирически изучены возможные источники некорректности поправки на ЭФ: специфика конкретных тестов, шкал, итераций измерений, индивидуально-психологических переменных (Ceci, Kanaya, 2010; Kaufman, 2010), гетерогенных и сравнительно небольших выборок валидизации в сравнении с выборками стандартизации (Zhu, Tulsky, 1999), нестабильность ЭФ в разных странах (Hagan et al., 2010а) и в выборках разных возрастов и уровня интеллекта (Sanborn, 2003; Zhou et al., 2010). Трудность систематического учета 
данных факторов приводит к заключению, что точность поправки оказывается спорной (Hagan et al., 2010b).

Подвижность пограничного критерия в оценке интеллекта составляет проблему и для таких областей практики в США, как определение права на получение социальных гарантий (Trahan, Stuebing, 2014) и годности к военной службе (Kanaya et al., 2003), где учет ЭФ не регламентирован. Все это подчеркивает важность изучения глубинных механизмов ЭФ.

\section{Заключение}

Наиболее важным для корректной интерпретации ЭФ является тот факт, что рост показателей интеллек- та не связан с приростом фактора g, a связан с дисперсией способностей, не входящей в g. В совокупности с данными о снижении мощности фактора g это означает, что происходит изменение структуры способностей в сторону их большей дифференциации и независимости друг от друга. Причины, стоящие за этими изменениями, скорее всего, разнообразны они могут проявляться одновременно и взаимодействовать между собой, они могут быть специфичными для разного уровня способностей и для разных исторических этапов. Знание особенностей и динамики ЭФ позволяет более профессионально и ответственно подходить к тестированию интеллектуальных способностей.

\section{Литература}

Батурин, Н. А. (2008). Современная психодиагностика в России. Вестник ЮУрГУ. Серия «Пихология», 32(2), 4-9.

Ушаков, Д. В. (2011). Психология интеллекта и одаренности. М.: Изд-во «Институт психологии $\mathrm{PAH}$.

Agrawal, N., Sinha, S. N., \& Jensen, A. R. (1984). Effects of inbreeding on Raven matrices. Behavior Genetics, 14(6), 579-585.

Beaujean, A. A., \& Osterlind, S. J. (2008). Using Item Response Theory to assess the Flynn Effect in the National Longitudinal Study of Youth 79 Children and Young Adults data. Intelligence, 36, 455-463. doi:10.1016/j.intell.2007.10.004

Beaujean, A. A., \& Sheng, Y. (2010). Examining the Flynn Effect in the General Social Survey Vocabulary test using item response theory. Personality and Individual Differences, 48(3), 294-298. doi:10.1016/j.paid.2009.10.019

Bocéréan, C., Fischer, J.-P., \& Flieller, A. (2003). Long-term comparison (1921-2001) of numerical knowledge in three to five-and-a-half year-old children. European Journal of Psychology of Education, 18, 405-424. doi:10.1007/BF03173244

Brand, C. R., Freshwater, S., \& Dockrell, W. B. (1989). Has there been a "massive" rise in IQ levels in the West? Evidence from Scottish children. The Irish Journal of Psychology, 10(3), 388-393. doi:10.1080/03033910.1989.10557756

Ceci, S. J., \& Kanaya, T. (2010). "Apples and oranges are both round": Furthering the discussion on the Flynn effect. Journal of Psychoeducational Assessment, 28, 441-447. doi:10.1177/ 0734282910373339 
Cunningham, M. D., \& Tassé, M. J. (2010). Looking to science rather than convention in adjusting IQ scores when death is at issue. Professional Psychology: Research and Practice, 41(5), 413-419. doi:10.1037/a0020226

Dickens, W. T., \& Flynn, J. R. (2001). Heritability estimates versus large environmental effects: the IQ paradox resolved. Psychological Review, 108(2), 346-369. doi:10.1037/0033-295X.108.2.346

Dutton, E., \& Lynn, R. (2013). A negative flynn effect in Finland, 1997-2009. Intelligence, 41(6), 817-820. doi:10.1016/j.intell.2013.05.008

Dutton, E., \& Lynn, R. (2015). A negative Flynn Effect in France, 1999 to 2008-9. Intelligence, 51, 67-70. doi:10.1016/j.intell.2015.05.005

Eppig, C., Fincher, C. L., \& Thornhill, R. (2010). Parasite prevalence and the worldwide distribution of cognitive ability. Proceedings of the Royal Society B: Biological Sciences, 277(1701), 3801-3808. doi:10.1098/rspb.2010.0973

Fitzgerald, S., Gray, N. S., \& Snowden, R. J. (2007). A comparison of WAIS-R and WAIS-III in the lower IQ range: Implications for learning disability diagnosis. Journal of Applied Research in Intellectual Disabilities, 20(2001), 323-330. doi:10.1111/j.1468-3148.2006.00349.x

Fletcher, J. M., Stuebing, K. K., \& Hughes, L. C. (2010). IQ scores should be corrected for the Flynn Effect in high-stakes decisions. Journal of Psychoeducational Assessment, 28, 469-473.

Flynn, J. R. (1984). The mean IQ of Americans: Massive gains 1932 to 1978. Psychological Bulletin, 95(1), 29-51. doi:10.1037/0033-2909.95.1.29

Flynn, J. R. (1987). Massive IQ gains in 14 nations: What IQ tests really measure. Psychological Bulletin, 101(2), 171-191.

Flynn, J. R. (2006). Tethering the elephant: Capital cases, IQ, and the Flynn effect. Psychology, Public Policy, and Law, 12(2), 170-189. doi:10.1037/1076-8971.12.2.170

Flynn, J. R. (2009). The WAIS-III and WAIS-IV: Daubert motions favor the certainly false over the approximately true. Applied Neuropsychology, 16, 98-104.

Flynn, J. R. (2015). The march of reason: What was hidden in our genes. In S. Goldstein, D. Princiotta, $\&$ J. A. Naglieri (Eds.), Handbook of intelligence: Evolutionary theory, historical perspective, and current concepts (pp. 471-485). New York: Springer.

Greenfield, P. M. (1998). The cultural elevation of IQ. In U. Neisser (Ed.), The rising curve (pp. 81-123). Washington, DC: American Psychological Association.

Gresham, F. M., \& Reschly, D. J. (2011). Standard of practice and flynn effect testimony in death penalty cases. Intellectual and Developmental Disabilities, 49(3), 131-140. doi:10.1352/1934-9556-49.3.131

Hagan, L. D., Drogin, E. Y., \& Guilmette, T. J. (2010a). IQ scores should not be adjusted for the Flynn Effect in capital punishment cases. Journal of Psychoeducational Assessment, 28, 474-476. doi:10.1177/073428291373343

Hagan, L. D., Drogin, E. Y., \& Guilmette, T. J. (2010b). Science rather than advocacy when reporting IQ scores. Professional Psychology: Research and Practice, 41(5), 420-423. doi:10.1037/a0021077

Herrnstein, R. J., \& Murray, C. (1994). Bell curve: Intelligence and class structure in American life. New York: Free Press.

Howard, R. W. (2001). Searching the real world for signs of rising population intelligence. Personality and Individual Differences, 30, 1039-1058. doi:10.1016/S0191-8869(00)00095-7

Husen, T., \& Tuijnman, A. (1991). The contribution of formal schooling to the increase in intellectual capital. Educational Researcher, 20(7), 17-25. doi:10.3102/0013189X020007017

Jensen, A. R. (1983). Effects of inbreeding on mental-ability factors. Personality and Individual Differences, 4(1), 71-87. doi:10.1038/266440a0 
Jensen, A. R. (1998). The g factor: The science of mental ability. Westport, CT: Praeger.

Juan-Espinosa, M., Cuevas, L., Escorial, S., \& García, L. F. (2006). The differentiation hypothesis and the Flynn effect. Psicothema, 18(1927), 284-287.

Kanaya, T., \& Ceci, S. J. (2012). The impact of the Flynn effect on LD diagnoses in special education. Journal of Learning Disabilities, 45(4), 319-326. doi:10.1177/0022219410392044

Kanaya, T., Scullin, M. H., \& Ceci, S. J. (2003). The Flynn effect and U.S. policies: the impact of rising IQ scores on American society via mental retardation diagnoses. The American Psychologist, 58(10), 778-790. doi:10.1037/0003-066X.58.10.778

Kane, H. D. (2000). A secular decline in Spearman's g: evidence from the WAIS, WAIS-R and WAIS-III. Personality and Individual Differences, 29(3), 561-566.

Kane, H. D., \& Oakland, T. D. (2000). Secular declines in Spearman's g: some evidence from the United States. The Journal of Genetic Psychology, 161, 337-345. doi:10.1080/00221320009596716

Kaufman, A. S. (2010). "In what way are apples and oranges alike?” A critique of Flynn's interpretation of the Flynn effect. Journal of Psychoeducational Assessment, 28(5), 382-398. doi:10.1177/0734282910373346

Lynn, R. (1982). IQ in Japan and the United States shows a growing disparity. Nature, 297, 222-223. doi:10.1038/297222a0

Lynn, R. (1990). The role of nutrition in secular increases in intelligence. Personality and Individual Differences, 11(3), 273-285. doi:10.1016/0191-8869(90)90241-I

Lynn, R. (2009). What has caused the Flynn effect? Secular increases in the Development Quotients of infants. Intelligence, 37(1), 16-24. doi:10.1016/j.intell.2008.07.008

Lynn, R., \& Cooper, C. (1993). A secular decline in Spearman's g in France. Learning and Individual Differences, 5(1), 43-48. doi:10.1016/1041-6080(93)90025-N

Lynn, R., \& Cooper, C. (1994). A secular decline in Spearman's g in Japan. Current Psychology, 13(1), 3-9.

Lynn, R., \& Harvey, J. (2008). The decline of the world's IQ. Intelligence, 36, 112-120. doi:10.1016/j.intell.2007.03.004

McGrew, K. S. (2015). Norm obsolescence: The Flynn Effect. In The death penalty and intellectual disability (pp. 155-169). Washington, DC: American Association on Intellectual and Developmental Disabilities.

Meisenberg, G., Lawless, E., Lambert, E., \& Newton, A. (2005). The Flynn effect in the Caribbean: Generational change in test performance in Domenica. Mankind Quarterly, 46, 29-70.

Merrill, M. A. (1938). The significance of IQ's on the revised Stanford-Binet scales. Journal of Educational Psychology, 29(9), 641-651.

Mingroni, M. A. (2007). Resolving the IQ paradox: heterosis as a cause of the Flynn effect and other trends. Psychological Review, 114(3), 806-829. doi:10.1037/0033-295X.114.3.806

Mingroni, M. A. (2014). Future efforts in Flynn effect research: Balancing reductionism with holism. Journal of Intelligence, 2(4), 122-155. doi:10.3390/jintelligence2040122

Must, O., \& Must, A. (2013). Changes in test-taking patterns over time. Intelligence, 41(6), 780-790. doi:10.1016/j.intell.2013.04.005

Neisser, U. (1997). Rising scores on intelligence tests. American Scientist, 85, 440-447.

Nevin, R. (2000). How lead exposure relates to temporal changes in IQ, violent crime, and unwed pregnancy. Environmental Research, 83(1), 1-22. doi:10.1006/enrs.1999.4045

Pietschnig, J., Tran, U. S., \& Voracek, M. (2013). Item-response theory modeling of IQ gains (the Flynn effect) on crystallized intelligence: Rodgers' hypothesis yes, Brand's hypothesis perhaps. Intelligence, 41(6), 791-801. doi:10.1016/j.intell.2013.06.005 
Pietschnig, J., \& Voracek, M. (2015). One century of global IQ gains: A formal meta-analysis of the Flynn effect (1909-2013). Perspectives on Psychological Science, 10(3), 282-306. doi:10.1177/ 1745691615577701

Rönnlund, M., \& Nilsson, L. G. (2009). Flynn effects on sub-factors of episodic and semantic memory: Parallel gains over time and the same set of determining factors. Neuropsychologia, 47, 2174-2180. doi:10.1016/j.neuropsychologia.2008.11.007

Rushton, J. P. (1985). Differential K theory: The sociobiology of individual and group differences. Personality and Individual Differences, 6(4), 441-452. doi:10.1016/0191-8869(85)90137-0

Rushton, J. P. (1999). Secular gains in IQ not related to the g factor and inbreeding depression - unlike Black-White differences: A reply to Flynn. Personality and Individual Differences, 26, 381-389.

Rushton, J. P., \& Jensen, A. R. (2010). The rise and fall of the Flynn effect as a reason to expect a narrowing of the Black-White IQ gap. Intelligence, 38(2), 213-219. doi:10.1016/j.intell.2009.12.002

Sanborn, K. J. (2003). Does the Flynn Effect differ by IQ level in samples of students classified as learning disabled? Journal of Psychoeducational Assessment, 21, 145-159. doi:10.1177/ 073428290302100203

Schooler, C. (1998). Environmental complexity and the Flynn effect. In U. Neisser (Ed.), The rising curve: Long-term gains in IQ and related measures (pp. 67-79). Washington, DC: American Psychological Association. doi:10.1159/000101067

Scullin, M. H. (2006). Large state-level fluctuations in mental retardation classifications related to introduction of renormed intelligence test. American Journal of Mental Retardation, 111(5), 322-335. doi:10.1352/0895-8017(2006)111[322:LSFIMR]2.0.CO;2

Shatz, S. M. (2008). IQ and fertility: A cross-national study. Intelligence, 36(2), 109-111. doi:10.1016/j.intell.2007.03.002

Shayer, M., \& Ginsburg, D. (2009). Thirty years on - a large anti-Flynn effect? (II): 13- and 14-yearolds. Piagetian tests of formal operations norms 1976-2006/7. The British Journal of Educational Psychology, 79, 409-418. doi:10.1348/978185408X383123

Shiu, W., Beaujean, A. A., Must, O., te Nijenhuis, J., \& Must, A. (2013). An item-level examination of the Flynn effect on the National Intelligence Test in Estonia. Intelligence, 41(6), 770-779. doi:10.1016/j.intell.2013.05.007

Storfer, M. (1999). Myopia, intelligence, and the expanding human neocortex: behavioral influences and evolutionary implications. The International Journal of Neuroscience, 98(3-4), 153-276. doi:10.3109/00207459908997465

Sundet, J. M., Barlaug, D. G., \& Torjussen, T. M. (2004). The end of the Flynn effect? A study of secular trends in mean intelligence test scores of Norwegian conscripts during half a century. Intelligence, 32, 349-362. doi:10.1016/j.intell.2004.06.004

Te Nijenhuis, J., Jongeneel-Grimen, B., \& Kirkegaard, E. O. W. (2014). Are Headstart gains on the g factor? A meta-analysis. Intelligence, 46, 209-215. doi:10.1016/j.intell.2014.07.001

Te Nijenhuis, J., \& van der Flier, H. (2013). Is the Flynn effect on g?: A meta-analysis. Intelligence, 41(6), 802-807. doi:10.1016/j.intell.2013.03.001

Te Nijenhuis, J., van Vianen, A. E. M., \& van der Flier, H. (2007). Score gains on g-loaded tests: No g. Intelligence, 35(3), 283-300. doi:10.1016/j.intell.2006.07.006

Teasdale, T. W., \& Owen, D. R. (2005). A long-term rise and recent decline in intelligence test performance: The Flynn Effect in reverse. Personality and Individual Differences, 39, 837-843. doi:10.1016/j.paid.2005.01.029 
Teasdale, T. W., \& Owen, D. R. (2008). Secular declines in cognitive test scores: A reversal of the Flynn Effect. Intelligence, 36, 121-126. doi:10.1016/j.intell.2007.01.007

Trahan, L., \& Stuebing, K. (2014). The Flynn effect: A meta-analysis. Psychological Bulletin, 140(5), 1332-1360. doi:10.1037/a0037173

Tuddenham, R. D. (1948). Soldier intelligence in World Wars I and II. The American Psychologist, 3, 54-56. doi:10.1037/h0054962

Williams, R. L. (2013). Overview of the Flynn effect. Intelligence, 41(6), 753-764. doi:10.1016/ j.intell.2013.04.010

Woodley, M. A. (2012a). A life history model of the Lynn-Flynn effect. Personality and Individual Differences, 53(2), 152-156. doi:10.1016/j.paid.2011.03.028

Woodley, M. A. (2012b). The social and scientific temporal correlates of genotypic intelligence and the Flynn effect. Intelligence, 40(2), 189-204. doi:10.1016/j.intell.2011.12.002

Woodley, M. A., te Nijenhuis, J., \& Murphy, R. (2013). Were the Victorians cleverer than us? The decline in general intelligence estimated from a meta-analysis of the slowing of simple reaction time. Intelligence, 41(6), 843-850. doi:10.1016/j.intell.2013.04.006

Woodley, M. A., te Nijenhuis, J., Must, O., \& Must, A. (2014). Controlling for increased guessing enhances the independence of the Flynn effect from g: The return of the Brand effect. Intelligence, 43(1), 27-34. doi:10.1016/j.intell.2013.12.004

Woodley of Menie, M. A. (2015). How fragile is our intellect? Estimating losses in general intelligence due to both selection and mutation accumulation. Personality and Individual Differences, 75, 80-84. doi:10.1016/j.paid.2014.10.047

Woodley of Menie, M. A., \& Fernandes, H. B. F. (2015). Do opposing secular trends on backwards and forwards digit span evidence the co-occurrence model? A comment on Gignac (2015). Intelligence, 50, 125-130. doi:10.1016/j.intell.2015.03.003

Young, G. W. (2012). A more intelligent and just Atkins: adjusting for the Flynn effect in capital determinations of mental retardation or intellectual disability. Venderbilt Law Reviere, 65, 615-675.

Zajonc, R. B., \& Mullally, P. R. (1997). Birth order: Reconciling conflicting effects. American Psychologist, 52(7), 685-699. doi:10.1037/0003-066X.52.7.685

Zhou, X., Zhu, J., \& Weiss, L. G. (2010). Peeking inside the "black box" of the Flynn Effect: Evidence from three Wechsler instruments. Journal of Psychoeducational Assessment, 28, 399-411. doi:10.1177/0734282910373340

Zhu, J., \& Tulsky, D. (1999). Can IQ gain be accurately quantified by a simple difference formula? Perceptual and Motor Skill, 88, 1255-1260.

Валуева Екатерина Александровна, научный сотрудник Института психологии РАН, ведущий научный сотрудник ГБОУ ВПО Московский городской психолого-педагогический университет, кандидат психологических наук Контакты: ekval@mail.ru

Белова Софъя Сергеевна - научный сотрудник Института психологии РАН, ведущий научный сотрудник ГБОУ ВПО Московский городской психологопедагогический университет, кандидат психологических наук.

Контакты: sbelova@gmail.com 


\title{
Flynn Effect: Contemporary Data Review
}

\author{
Ekaterina A. Valueva \\ Research fellow, Institute of Psychology of Russian Academy of Sciences; \\ Senior research fellow, Moscow State University of Psychology \& Education, Ph.D. \\ E-mail: ekval@mail.ru \\ Sofya S. Belova \\ Research fellow, Institute of Psychology of Russian Academy of Sciences; \\ Senior research fellow, Moscow State University of Psychology \& Education, Ph.D. \\ E-mail: sbelova@gmail.com \\ Address: * 13 build. 1 Yaroslavskaya str., Moscow, 129366, Russian Federation \\ ** 29 Sretenka str., Moscow, 127051, Russian Federation
}

\begin{abstract}
The article presents the review of contemporary literature on the problem of the Flynn effect (FE). The FE is the increase of intelligence tests scores throughout the XX century with the rate of approximately 3 IQ points per decade. The article describes the essence of the FE and methods of its measurement, metaanalytical data on the size and stability of the FE between populations and in time. Authors also considered the manifestation of the FE in cognitive tasks different from intelligence tests, and in the domain of intellectual achievements. The special attention is devoted to the discussion of the causes of the FE. The most popular hypotheses are the environmental explanations of the FE (improvement of nutrition, changes in the family structure, growth in quantity and quality of cognitive stimulation, etc.). Along with that, there are explanations which connect the FE with genetic changes, as well as with artifacts that appear in the process of intelligence testing. It is also debated whether the FE depicts the real increase in intelligence in people. The data is provided that shows that the FE is more pronounced in tasks that have lowest loadings on the general factor of intelligence (g factor). At the same time there is data that prove the decrease in genotypic intelligence. It is concluded that the FE is associated with the change in structure of cognitive abilities and acquisition of problem solving skills, which capture rules and abstract reasoning operations. Thus the FE masks the tendency of decrease in genotypic intelligence. In the conclusion of the article the practical consequences of the FE in the sphere of education, forensic practice and social policies are analyzed.
\end{abstract}

Keywords: Flynn effect, intelligence, intelligence tests.

\section{References}

Agrawal, N., Sinha, S. N., \& Jensen, A. R. (1984). Effects of inbreeding on Raven matrices. Behavior Genetics, 14(6), 579-585.

Baturin, N. A. (2008). Sovremennaya psikhodiagnostika v Rossii [Contemporary psychodiagnostics in Russia]. Bulletin of the South Ural State University. Series "Psychology", 32(2), 4-9. 
Beaujean, A. A., \& Osterlind, S. J. (2008). Using Item Response Theory to assess the Flynn Effect in the National Longitudinal Study of Youth 79 Children and Young Adults data. Intelligence, 36, 455-463. doi:10.1016/j.intell.2007.10.004

Beaujean, A. A., \& Sheng, Y. (2010). Examining the Flynn Effect in the General Social Survey Vocabulary test using item response theory. Personality and Individual Differences, 48(3), 294-298. doi:10.1016/j.paid.2009.10.019

Bocéréan, C., Fischer, J.-P., \& Flieller, A. (2003). Long-term comparison (1921-2001) of numerical knowledge in three to five-and-a-half year-old children. European Journal of Psychology of Education, 18, 405-424. doi:10.1007/BF03173244

Brand, C. R., Freshwater, S., \& Dockrell, W. B. (1989). Has there been a "massive" rise in IQ levels in the West? Evidence from Scottish children. The Irish Journal of Psychology, 10(3), 388-393. doi:10.1080/03033910.1989.10557756

Ceci, S. J., \& Kanaya, T. (2010). "Apples and oranges are both round": Furthering the discussion on the Flynn effect. Journal of Psychoeducational Assessment, 28, 441-447. doi:10.1177/ 0734282910373339

Cunningham, M. D., \& Tassé, M. J. (2010). Looking to science rather than convention in adjusting IQ scores when death is at issue. Professional Psychology: Research and Practice, 41(5), 413-419. doi:10.1037/a0020226

Dickens, W. T., \& Flynn, J. R. (2001). Heritability estimates versus large environmental effects: the IQ paradox resolved. Psychological Review, 108(2), 346-369. doi:10.1037/0033-295X.108.2.346

Dutton, E., \& Lynn, R. (2013). A negative flynn effect in Finland, 1997-2009. Intelligence, 41(6), 817-820. doi:10.1016/j.intell.2013.05.008

Dutton, E., \& Lynn, R. (2015). A negative Flynn Effect in France, 1999 to 2008-9. Intelligence, 51, 67-70. doi:10.1016/j.intell.2015.05.005

Eppig, C., Fincher, C. L., \& Thornhill, R. (2010). Parasite prevalence and the worldwide distribution of cognitive ability. Proceedings of the Royal Society B: Biological Sciences, 277(1701), 3801-3808. doi:10.1098/rspb.2010.0973

Fitzgerald, S., Gray, N. S., \& Snowden, R. J. (2007). A comparison of WAIS-R and WAIS-III in the lower IQ range: Implications for learning disability diagnosis. Journal of Applied Research in Intellectual Disabilities, 20(2001), 323-330. doi:10.1111/j.1468-3148.2006.00349.x

Fletcher, J. M., Stuebing, K. K., \& Hughes, L. C. (2010). IQ scores should be corrected for the Flynn Effect in high-stakes decisions. Journal of Psychoeducational Assessment, 28, 469-473.

Flynn, J. R. (1984). The mean IQ of Americans: Massive gains 1932 to 1978. Psychological Bulletin, 95(1), 29-51. doi:10.1037/0033-2909.95.1.29

Flynn, J. R. (1987). Massive IQ gains in 14 nations: What IQ tests really measure. Psychological Bulletin, 101(2), 171-191.

Flynn, J. R. (2006). Tethering the elephant: Capital cases, IQ, and the Flynn effect. Psychology, Public Policy, and Law, 12(2), 170-189. doi:10.1037/1076-8971.12.2.170

Flynn, J. R. (2009). The WAIS-III and WAIS-IV: Daubert motions favor the certainly false over the approximately true. Applied Neuropsychology, 16, 98-104.

Flynn, J. R. (2015). The march of reason: What was hidden in our genes. In S. Goldstein, D. Princiotta, \& J. A. Naglieri (Eds.), Handbook of intelligence: Evolutionary theory, historical perspective, and current concepts (pp. 471-485). New York: Springer.

Greenfield, P. M. (1998). The cultural elevation of IQ. In U. Neisser (Ed.), The rising curve (pp. 81-123). Washington, DC: American Psychological Association. 
Gresham, F. M., \& Reschly, D. J. (2011). Standard of practice and flynn effect testimony in death penalty cases. Intellectual and Developmental Disabilities, 49(3), 131-140. doi:10.1352/1934-9556-49.3.131

Hagan, L. D., Drogin, E. Y., \& Guilmette, T. J. (2010a). IQ scores should not be adjusted for the Flynn Effect in capital punishment cases. Journal of Psychoeducational Assessment, 28, 474-476. Hagan, L. D., Drogin, E. Y., \& Guilmette, T. J. (2010b). Science rather than advocacy when reporting IQ scores. Professional Psychology: Research and Practice, 41(5), 420-423. doi:10.1037/a0021077

Herrnstein, R. J., \& Murray, C. (1994). Bell curve: Intelligence and class structure in American life. New York: Free Press.

Howard, R. W. (2001). Searching the real world for signs of rising population intelligence. Personality and Individual Differences, 30, 1039-1058. doi:10.1016/S0191-8869(00)00095-7

Husen, T., \& Tuijnman, A. (1991). The contribution of formal schooling to the increase in intellectual capital. Educational Researcher, 20(7), 17-25. doi:10.3102/0013189X020007017

Jensen, A. R. (1983). Effects of inbreeding on mental-ability factors. Personality and Individual Differences, 4(1), 71-87. doi:10.1038/266440a0

Jensen, A. R. (1998). The g factor: The science of mental ability. Westport, CT: Praeger.

Juan-Espinosa, M., Cuevas, L., Escorial, S., \& García, L. F. (2006). The differentiation hypothesis and the Flynn effect. Psicothema, 18(1927), 284-287.

Kanaya, T., \& Ceci, S. J. (2012). The impact of the Flynn effect on LD diagnoses in special education. Journal of Learning Disabilities, 45(4), 319-326. doi:10.1177/0022219410392044

Kanaya, T., Scullin, M. H., \& Ceci, S. J. (2003). The Flynn effect and U.S. policies: the impact of rising IQ scores on American society via mental retardation diagnoses. The American Psychologist, 58(10), 778-790. doi:10.1037/0003-066X.58.10.778

Kane, H. D. (2000). A secular decline in Spearman's g: evidence from the WAIS, WAIS-R and WAIS-III. Personality and Individual Differences, 29(3), 561-566.

Kane, H. D., \& Oakland, T. D. (2000). Secular declines in Spearman's g: some evidence from the United States. The Journal of Genetic Psychology, 161, 337-345. doi:10.1080/00221320009596716

Kaufman, A. S. (2010). "In what way are apples and oranges alike?” A critique of Flynn's interpretation of the Flynn effect.Journal of Psychoeducational Assessment, 28(5), 382-398. Lynn, R. (1982). IQ in Japan and the United States shows a growing disparity. Nature, 297, 222-223. Lynn, R. (1990). The role of nutrition in secular increases in intelligence. Personality and Individual Differences, 11(3), 273-285. doi:10.1016/0191-8869(90)90241-I

Lynn, R. (2009). What has caused the Flynn effect? Secular increases in the Development Quotients of infants. Intelligence, 37(1), 16-24. doi:10.1016/j.intell.2008.07.008

Lynn, R., \& Cooper, C. (1993). A secular decline in Spearman's g in France. Learning and Individual Differences, 5(1), 43-48. doi:10.1016/1041-6080(93)90025-N

Lynn, R., \& Cooper, C. (1994). A secular decline in Spearman's g in Japan. Current Psychology, 13(1), 3-9.

Lynn, R., \& Harvey, J. (2008). The decline of the world's IQ. Intelligence, 36, 112-120. doi:10.1016/j.intell.2007.03.004

McGrew, K. S. (2015). Norm obsolescence: The Flynn Effect. In The death penalty and intellectual disability (pp. 155-169). Washington, DC: American Association on Intellectual and Developmental Disabilities.

Meisenberg, G., Lawless, E., Lambert, E., \& Newton, A. (2005). The Flynn effect in the Caribbean: Generational change in test performance in Domenica. Mankind Quarterly, 46, 29-70.

Merrill, M. A. (1938). The significance of IQ's on the revised Stanford-Binet scales. Journal of Educational Psychology, 29(9), 641-651. 
Mingroni, M. A. (2007). Resolving the IQ paradox: heterosis as a cause of the Flynn effect and other trends. Psychological Reviere, 114(3), 806-829. doi:10.1037/0033-295X.114.3.806

Mingroni, M. A. (2014). Future efforts in Flynn effect research: Balancing reductionism with holism. Journal of Intelligence, 2(4), 122-155. doi:10.3390/jintelligence2040122

Must, O., \& Must, A. (2013). Changes in test-taking patterns over time. Intelligence, 41(6), 780-790. doi:10.1016/j.intell.2013.04.005

Neisser, U. (1997). Rising scores on intelligence tests. American Scientist, 85, 440-447.

Nevin, R. (2000). How lead exposure relates to temporal changes in IQ, violent crime, and unwed pregnancy. Environmental Research, 83(1), 1-22. doi:10.1006/enrs.1999.4045

Pietschnig, J., Tran, U. S., \& Voracek, M. (2013). Item-response theory modeling of IQ gains (the Flynn effect) on crystallized intelligence: Rodgers' hypothesis yes, Brand's hypothesis perhaps. Intelligence, 41(6), 791-801. doi:10.1016/j.intell.2013.06.005

Pietschnig, J., \& Voracek, M. (2015). One century of global IQ gains: A formal meta-analysis of the Flynn effect (1909-2013). Perspectives on Psychological Science, 10(3), 282-306. Rönnlund, M., \& Nilsson, L. G. (2009). Flynn effects on sub-factors of episodic and semantic memory: Parallel gains over time and the same set of determining factors. Neuropsychologia, 47, 2174-2180. doi:10.1016/j.neuropsychologia.2008.11.007

Rushton, J. P. (1985). Differential K theory: The sociobiology of individual and group differences. Personality and Individual Differences, 6(4), 441-452. doi:10.1016/0191-8869(85)90137-0

Rushton, J. P. (1999). Secular gains in IQ not related to the g factor and inbreeding depression - unlike Black-White differences: A reply to Flynn. Personality and Individual Differences, 26, 381-389.

Rushton, J. P., \& Jensen, A. R. (2010). The rise and fall of the Flynn effect as a reason to expect a narrowing of the Black-White IQ gap. Intelligence, 38(2), 213-219. doi:10.1016/j.intell.2009.12.002

Sanborn, K. J. (2003). Does the Flynn Effect differ by IQ level in samples of students classified as learning disabled? Journal of Psychoeducational Assessment, 21, 145-159. doi:10.1177/ 073428290302100203

Schooler, C. (1998). Environmental complexity and the Flynn effect. In U. Neisser (Ed.), The rising curve: Long-term gains in IQ and related measures (pp. 67-79). Washington, DC: American Psychological Association. doi:10.1159/000101067

Scullin, M. H. (2006). Large state-level fluctuations in mental retardation classifications related to introduction of renormed intelligence test. American Journal of Mental Retardation, 111(5), 322-335. doi:10.1352/0895-8017(2006)111[322:LSFIMR]2.0.CO;2

Shatz, S. M. (2008). IQ and fertility: A cross-national study. Intelligence, 36(2), 109-111. doi:10.1016/j.intell.2007.03.002

Shayer, M., \& Ginsburg, D. (2009). Thirty years on - a large anti-Flynn effect? (II): 13- and 14-yearolds. Piagetian tests of formal operations norms 1976-2006/7. The British Journal of Educational Psychology, 79, 409-418. doi:10.1348/978185408X383123

Shiu, W., Beaujean, A. A., Must, O., te Nijenhuis, J., \& Must, A. (2013). An item-level examination of the Flynn effect on the National Intelligence Test in Estonia. Intelligence, 41(6), 770-779. doi:10.1016/j.intell.2013.05.007

Storfer, M. (1999). Myopia, intelligence, and the expanding human neocortex: behavioral influences and evolutionary implications. The International Journal of Neuroscience, 98(3-4), 153-276. Sundet, J. M., Barlaug, D. G., \& Torjussen, T. M. (2004). The end of the Flynn effect? A study of secular trends in mean intelligence test scores of Norwegian conscripts during half a century. Intelligence, 32, 349-362. doi:10.1016/j.intell.2004.06.004 
Te Nijenhuis, J., Jongeneel-Grimen, B., \& Kirkegaard, E. O. W. (2014). Are Headstart gains on the g factor? A meta-analysis. Intelligence, 46, 209-215. doi:10.1016/j.intell.2014.07.001

Te Nijenhuis, J., \& van der Flier, H. (2013). Is the Flynn effect on g?: A meta-analysis. Intelligence, 41(6), 802-807. doi:10.1016/j.intell.2013.03.001

Te Nijenhuis, J., van Vianen, A. E. M., \& van der Flier, H. (2007). Score gains on g-loaded tests: No g. Intelligence, 35(3), 283-300. doi:10.1016/j.intell.2006.07.006

Teasdale, T. W., \& Owen, D. R. (2005). A long-term rise and recent decline in intelligence test performance: The Flynn Effect in reverse. Personality and Individual Differences, 39, 837-843. doi:10.1016/j.paid.2005.01.029

Teasdale, T. W., \& Owen, D. R. (2008). Secular declines in cognitive test scores: A reversal of the Flynn Effect. Intelligence, 36, 121-126. doi:10.1016/j.intell.2007.01.007

Trahan, L., \& Stuebing, K. (2014). The Flynn effect: A meta-analysis. Psychological Bulletin, 140(5), 1332-1360. doi:10.1037/a0037173

Tuddenham, R. D. (1948). Soldier intelligence in World Wars I and II. The American Psychologist, 3, 54-56. doi:10.1037/h0054962

Ushakov, D. V. (2011). Psikhologiya intellekta i odarennosti [The psychology of intelligence and giftedness]. Moscow: The Institute of Psichology of the Russian Academy of Sciences.

Williams, R. L. (2013). Overview of the Flynn effect. Intelligence, 41(6), 753-764. doi:10.1016/ j.intell.2013.04.010

Woodley, M. A. (2012a). A life history model of the Lynn-Flynn effect. Personality and Individual Differences, 53(2), 152-156. doi:10.1016/j.paid.2011.03.028

Woodley, M. A. (2012b). The social and scientific temporal correlates of genotypic intelligence and the Flynn effect. Intelligence, 40(2), 189-204. doi:10.1016/j.intell.2011.12.002

Woodley, M. A., te Nijenhuis, J., \& Murphy, R. (2013). Were the Victorians cleverer than us? The decline in general intelligence estimated from a meta-analysis of the slowing of simple reaction time. Intelligence, 41(6), 843-850. doi:10.1016/j.intell.2013.04.006

Woodley, M. A., te Nijenhuis, J., Must, O., \& Must, A. (2014). Controlling for increased guessing enhances the independence of the Flynn effect from g: The return of the Brand effect. Intelligence, 43(1), 27-34. doi:10.1016/j.intell.2013.12.004

Woodley of Menie, M. A. (2015). How fragile is our intellect? Estimating losses in general intelligence due to both selection and mutation accumulation. Personality and Individual Differences, 75, 80-84. doi:10.1016/j.paid.2014.10.047

Woodley of Menie, M. A., \& Fernandes, H. B. F. (2015). Do opposing secular trends on backwards and forwards digit span evidence the co-occurrence model? A comment on Gignac (2015). Intelligence, 50, 125-130. doi:10.1016/j.intell.2015.03.003

Young, G. W. (2012). A more intelligent and just Atkins: adjusting for the Flynn effect in capital determinations of mental retardation or intellectual disability. Venderbilt Law Review, 65, 615-675.

Zajonc, R. B., \& Mullally, P. R. (1997). Birth order: Reconciling conflicting effects. American Psychologist, 52(7), 685-699. doi:10.1037/0003-066X.52.7.685

Zhou, X., Zhu, J., \& Weiss, L. G. (2010). Peeking inside the "black box" of the Flynn Effect: Evidence from three Wechsler instruments. Journal of Psychoeducational Assessment, 28, 399-411. doi:10.1177/0734282910373340

Zhu, J., \& Tulsky, D. (1999). Can IQ gain be accurately quantified by a simple difference formula? Perceptual and Motor Skill, 88, 1255-1260. 